\title{
A brief introduction to the new architecture of SIETTE
}

\author{
Eduardo Guzmán, Ricardo Conejo \\ Departamento de Lenguajes y Ciencias de la Computación. \\ E.T.S.I. Informática. Bulevar Louis Pasteur, 35. 29071, Málaga. SPAIN \\ \{guzman,conejo\}@lcc.uma.es
}

\begin{abstract}
SIETTE is a web-based adaptive testing system released some years ago. It implements Computerized Adaptive Tests. In these tests the selection of the questions posed to students, the decision to finalize the test is accomplished adaptively. In this paper, we present the new architecture of SIETTE and some new features recently implemented.
\end{abstract}

\section{Introduction}

Systems that implement test-based assessment usually use heuristic-based techniques. However, there is another kind of tests, the Computerized Adaptive Tests (CAT) [7], which are based on a theory with underlying theoretical basis. This theory defines which questions (called items) must be posed to students, when the tests must finish, and how the student knowledge can be inferred from students' performance during the test. To this end, CATs use an underlying psychometric theory called Item Response Theory (IRT) [3]. In contrast to other testing assessment mechanisms, IRT ensures that obtained student knowledge estimations do not depend on the items used in the estimation process.

SIETTE is a web-based adaptive system for CAT generation and elicitation. In 1998, the first version of this system was developed [6]. However, from 2000 until these days, the second version of SIETTE has been re-implemented from scratch. A fully working version is available at http://www.lcc.uma.es/siette. A subject called "Demo" has been defined to show the new features of SIETTE.

It keeps the virtues of the preceding version, improves most of its drawbacks, and has added some other new features. The new implementation has been carried out because the previous architecture hindered the introduction of the new features.

In this paper, we provide a global snapshot of the new architecture of SIETTE, and some of the improvements introduced in the student classroom module.

\section{The architecture of SIETTE}

SIETTE allows CAT construction and delivering through web interfaces. It can work as a standalone assessment tool or inside other web-based adaptive systems, as a student knowledge diagnosis tool. It is a multilingual system, currently translated to 
Spanish and English, but open to include other languages. Tests managed by SIETTE can be adaptive, but heuristic-based conventional tests can be also constructed. Fig. 1 collects the new architecture of the system. It comprises two main parts: the student workspace and the authoring environment.

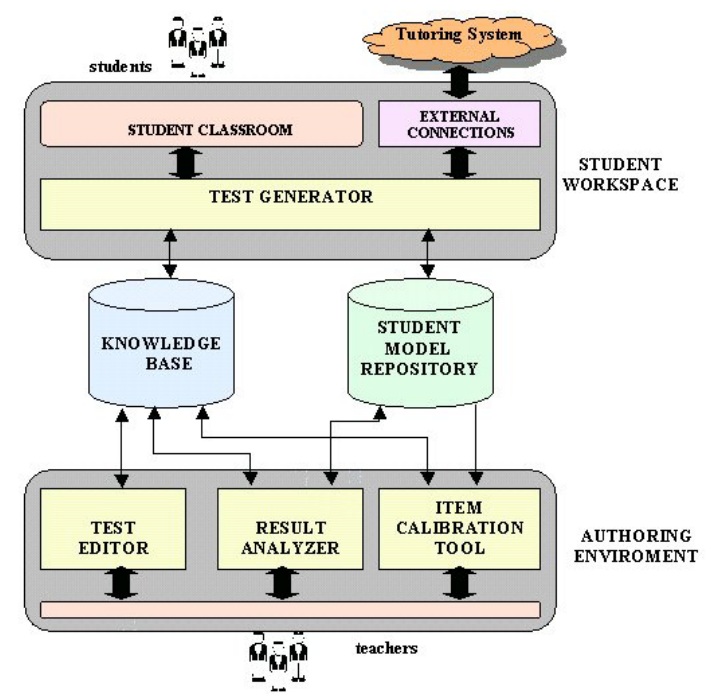

Fig. 1. Architecture of SIETTE

The knowledge base: It is where items, tests and topics are stored. All these contents are structured in subjects. Each subject comprises a set of topics. In turn, each topic can be decomposed into a set of subtopics following aggregation relations. Accordingly, all topics of a subject can be seen as a granularity hierarchy [5]. Items are associated to topics in such a way that if an item is related to a topic, it means it can be used to assess the topic and any parent of that topic. Tests are defined on the topics they assess, the rules for selecting items and the finalization criterion.

The student model repository: It stores the learner models of those that have taken a test. Learner models are probabilistic estimations of the students' knowledge is the topics of tests taken. Additionally, the students answers are also stored for statistical analysis and item calibration.

The student workspace: This is where students take tests. The main component of this module is the test generator. It delivers CATs that are suited to students' needs. Two interfaces can be used to access to generated tests:

- Student classroom: Here, students can take tests for self-assessment, and teachers can administer tests for grading.

- Interface for external connections: This interface permits SIETTE to work as a diagnosis tool in other web-based adaptive hypermedia educational systems. An propietary simple protocol [4] has been defined to this purpose.

The authoring environment: It is composed by a set of tools used by teachers. They allow content creation and update, as well as analyzing the performances of students that have taken tests, and the behavior of items used. It comprises: 
- The test editor: Through this tool, teachers can define topics and subtopics, and introduce items of different kinds related to each topic or subtopic. They can also define different tests by given the rules to be used.

- The result analyzer: This tool presents graphically the data collected from the students' performance, like the number of students that have given a certain answer, etc.

- The item calibration tool: In CATs, items are characterized by means of probabilistic functions, the Item Characteristic Curves (ICCs). Each one of these functions predicts the behavior of students that answer the corresponding item, and are determined by a set of parameters. These parameters are inferred by calibration techniques [1]. Currently, this module is an under development.

\section{The student classroom}

In adaptive testing, when a student takes a test, he will be administered items one by one in terms of his knowledge level current estimation. The item selected to be administered is the one which will make the student's knowledge estimation more accurate. After the student answers the item, his knowledge level is estimated taking the response into account. This process is carried out until his knowledge estimation is accurate enough.

In SIETTE, student must provide a username and a password to access the tests. Although there are tests restricted to predefined sets of students, others can be freely accessed by simply supplying some optional personal information (e.g. name, surname, email, etc.). Students can be organized in groups defined by teachers. When the student has been authenticated, his personalized list of subjects is displayed. Three types of users profiles [4] are managed in SIETTE: teachers, learners and examinees. In terms of the student profile, the test generator adapts the test as follows:

- Teachers: They are responsible of test creation. Through this profile, they can have a look at their tests before any student takes them. Consequently, when a teacher accesses to the test generator tool, all the tests of his subjects (or the subjects on which he has privileges) are available.

- Learners: They are students that take tests for self-assessment. In this kind of tests, item correction is shown immediately after the student answers. These tests are administered with item hints and feedbacks.

- Examinees: They are students that take tests for academic grading. Item correction might be shown at the end or the test (or never). No item hints or feedbacks are provided.

\section{Conclusions}

A new version of SIETTE has been released. It is base upon a well-founded theory and generates CATs for grading or self-assessment. New features modify the adaptive behavior of Siette for the item selection, student assessment and the test finalization 
criteria. These criteria are based on the performance of the students while taking the tests. Furthermore SIETTE incorporates some adaptable features to the user profile, and can presents the test differently to teachers; to students that take the test for selfassessment (providing item correction, feedbacks and hints), and to those that take the test for grading.

\section{Acknowledge and Disclaimer}

SIETTE is going to be used as a part of the project LeActiveMath, funded under FP6 (Contract. $\mathrm{N}^{\circ}$ 507826). The content of any publication of any kind produced by the LeActiveMath consortium is the sole responsibility of its authors and in no way represents the views of the EC and its services who cannot be held responsible for any use that might be made of data appearing therein..

\section{References}

1. Glas, C.A.W. Item calibration and parameter drift. In Van der Linden, W.J. and Glas, C.A.W. (eds.) Computerized Adaptive Testing: Theory and Practice. Kluwer Academic Publisher,(2000).

2. Guzmán, E. and Conejo, R. An adaptive assessment tool integrable into Internet-based learning systems. Méndez Vilas, A., Mesa González, J. A., and Solo de Zaldívar, I. Educational Technology: International Conference on TIC's in Education. I, pp. 139-143. 2002. Sociedad de la Información.

3. Hambleton, R.K., Swaminathan, J., and Rogers, H.J. Fundamentals of Item Response Theory. Sage publications, California, USA.

4. Kay, J. Stereotypes, Student Models and Scrutability.Proceedings of the International Conference on Intelligent Tutoring Systems (ITS 2000). (2000).

5. McCalla, G. I. and Greer, J. E. Granularity-Based Reasoning and Belief Revision in Student Models. Greer, J. E. and McCalla, G. Student Modeling: The Key to Individualized Knowledge-Based Instruction. 125, 39-62. 94. Berlin Heidelberg, Springer Verlag. NATO ASI Series.

6. Ríos, A., Millán, E., Trella, M., Pérez-de-la-Cruz, J. L., and Conejo, R. Internet Based Evaluation System. Lajoie, S. and Vivet, M. Open Learning Environments: New Computational Technologies to Support Learning, Exploration and Collaboration. Proceedings of the 9th World Conference of Artificial Intelligence and Education AIED'99. 387-394. 99. Amsterdam, IOS Press. (1999)

7. van der Linden, W.J. and Glas, C.A.W. Computerized Adaptive Testing: Theory and Practice. Kluwer Academic Publishers, Netherlands (2000). 